\section{Prediction and screening of esophageal varices in cirrhotic patients using doppler US hemodynamic indices of portal system}

\author{
Ali Hekmatnia, ${ }^{1}$ Rozbeh Barikbin, ${ }^{1}$ \\ Maryam Farghadani, ${ }^{1}$ Navid Omidifar, ${ }^{1}$ \\ Peyman Adibi ${ }^{2}$ \\ ${ }^{1}$ Department of Radiology; ${ }^{2}$ Liver \\ Diseases Research Center, Isfahan \\ University of Medical Sciences, Iran
}

\section{Abstract}

It is currently recommended that all patients with liver cirrhosis undergo upper gastrointestinal endoscopy (UGIE) to identify those who have esophageal varices (EV) that carry a high risk of bleeding and may benefit from prophylactic measures. In the future, this social and medical burden will increase due to the greater number of patients with chronic liver disease and their improved survival. The aim of this study was to assess the value of Doppler sonography for the prediction/screening of EV)in cirrhotic patients. In this twoyear prospective study, 50 patients with liver cirrhosis, referred to Al-Zahra hospital, were enrolled. Patients underwent detailed clinical examination, biochemistry tests (hematology, liver function tests), Gray-scale ultrasonography and Doppler sonography of hepatoportal system. Degree of esophageal varices was assessed at UGIE; Paquet's grades 0 - III were classified as group A (0-I; No or Mild EV) and group B (II-III; Moderate to severe EV). Three portal hemodynamic indices including liver vascular index, portal hypertension index, and congestive index was also measured. Degree of esophageal varices was assessed at UGIE. The relationship between the presence and degree of EVs with Doppler results were evaluated. Among 50 consecutive cirrhotic patients (41 males and 9 female, with mean age of $52.1( \pm 16.2)$ year) were enrolled. 19 (38\%) patients were placed in group A (No or mild EV), while 31(62\%) had endoscopic evidences of moderate to severe esophageal varices (group B). Our study showed that among three portal hemodynamic indices we studied, only portal hypertension index has statistically significant correlation with degree of EVs confirmed by UGIE ( $\mathrm{P}=0.029)$. Doppler ROC area under the curve was not significant but was near to be $(\mathrm{AUC}=0.64)$. Our data indicate that Doppler sonographic evaluations have no highly accurate predicting value for the presence of $\mathrm{EV}$ and its severity in patients with cirrhosis. However, we think Doppler study can be helpful for further investigation and finding more established and unchangeable information.

\section{Introduction}

Esophageal varix (EV) is a common major complication in patients with cirrhosis, with an estimated prevalence about $35-80 \%{ }^{1}$ and an annual incidence of approximately $5 \%$ for EV formation in patients with liver cirrhosis. ${ }^{2,3}$ The presence of varices correlates with the severity of liver diseases; varices are present in $30-40 \%$ of patients with compensated cirrhosis (Child-Pugh class A) and in $60-85 \%$ of patients with decompensated cirrhosis (ChildPugh classes B and C). ${ }^{4,5}$ Esophageal varices are the most common sources of bleeding in patients with cirrhosis. ${ }^{6}$ It is considerable that esophageal variceal bleeding might be a lethal complication in these patients with estimated mortality rate of $20-5 \% .5-8$ Variceal bleeding occurs in approximately one third of the patients with $\mathrm{EV}$, which may be repeated in up to $70 \%$ of these cases. ${ }^{9,10}$ Endoscopic screening for esophageal varices is recommended in patients with newly diagnosed chronic cirrhosis, because medical treatment must be considered as soon as varices are detected to prevent bleeding ${ }^{11}$ Some recent studies have evaluated different noninvasive measures, such as capsule endoscopy, biochemical, clinical and ultrasonographic parameters to predict the presence of esophageal varices before invasive screening endoscopy. ${ }^{12-15}$ Use of accurate and specific non-invasive methods may help to identify high-risk patients for esophageal varix development who can benefit from prophylactic pharmacologic and endoscopic therapies and to avoid unnecessary endoscopy in lowrisk patients. ${ }^{16}$ Some new studies have suggested using the ultrasonographic examination as a simple, inexpensive, accurate, and noninvasive technique. Various ultrasonographic (US) indexes, including spleen size, portal vein velocity (PVV), portal vein diameter, hepatic impedance indexes, splenic impedance indexes, and results of multi-detector computed tomographic esophagography, have been shown to be predictive of the severity of esophageal varices or risks of variceal bleeding in patients with cirrhosis. ${ }^{17-20}$ However, no consistent results have been reported yet.

The purpose of our study was to assess the value of US indexes for predicting the presence of esophageal varices in patients with cirrhosis. We have used endoscopy as the reference standard.
Correspondence: Peyman Adibi, Liver Diseases Research Center, Isfahan University of Medical Sciences, Iran. E-mail: adibi@med.mui.ac.ir

Key words: liver cirrhosis, esophageal varices, doppler sonography, spleen diameter, screening, liver vascular index, portal hypertensive index congestive index.

Received for publication: 20 May 2011.

Revision received: 7 July 2011.

Accepted for publication: 10 August 2011.

This work is licensed under a Creative Commons Attribution NonCommercial 3.0 License (CC BYNC 3.0).

(C) Copyright A. Hekmatnia et al., 2011

Licensee PAGEPress, Italy

Gastroenterology Insights 2011; 3:e4

doi:10.4081/gi.2011.e4

\section{Materials and Methods}

We enrolled 50 of consecutive newly diagnosed cirrhotic patients who were visited at our clinic, prior to any treatment in the $\mathrm{Al}$ Zahra hospital (affiliated to the Isfahan University of Medical Sciences). The diagnosis of cirrhosis was based on a liver biopsy or clinical and biochemical evaluations.

The following information was taken from each patient: age, gender, etiology of cirrhosis, onset of the disease, biochemical parameters [aspartate amino-transferase (SGOT), alanine amino-transferase (SGPT), total bilirubin, serum albumin, prothrombin activity (\%), International ratio (INR), and serum creatinine), platelet count, presence and degree of ascites, and encephalopathy assessed based on Child-Pugh's criteria. ${ }^{13}$

Patients with mentioned criteria were excluded from the study: those on diuretic or vasoactive treatment, those with previous gastrointestinal bleeding, hepatorenal syndrome during the past 3 month, hepatocellular carcinoma, any history of thrombosis or splenectomy due to any reason, thrombocytosis (platelet count $>450000 \mathrm{~N} / \mathrm{mm}^{3}$ ), current alcohol intake, previous surgery for portal hypertension or trans-jugular intra-hepatic portosystemic stent shunt placement, and clear signs of portal hypertension (ascites or hepatic encephalopathy).

All patients underwent color Doppler ultrasonic examination after endoscopy by a radiologist blinded to the results of endoscopy. They were evaluated for the presence and grade of $\mathrm{EV}$, and portal hypertension evidences. In the presence of EV, its size was graded as 0-III using the Paquet's grading system. ${ }^{13}$ Then patients were classified either as having mod- 
erate to severe EVs (grades II-III; Group B) or having mild or no varices (grades 0 or I; Group A). All patients were kept fasting for at least $6 \mathrm{hrs}$ prior to the ultrasonography. As well, the main Doppler factors were always taken by the same equipment (with a 3.5-5 $\mathrm{MHz}$ curve array transducer, G-50 Siemens) and by the same operator: i) Portal vein flow velocity as time average maximal velocity in $\mathrm{cm} / \mathrm{s}$ and portal vein diameter; ii) hepatic artery resistance index (RI) measured in the intrahepatic main branches $[\mathrm{RI}=$ (systolic velocity - end diastolic velocity)/systolic velocity]; iii) splenic artery RI measured intra-parenchymally near to hilum; iv) spleen size (length of its longest axis); and v) presence of portal-systemic collaterals.

The following indices were calculated: i) The liver vascular index as the ratio of portal venous velocity to hepatic arterial resistance index; ii) congestion index (CI) of the portal vein with dividing portal vein cross-sectional area by portal blood velocity; and iii) portal hypertensive index as (hepatic artery $\left.\mathrm{RI}^{*} 0.69\right)^{*}$ (splenic artery $\mathrm{R}^{*} 0.87$ )/portal vein mean velocity.

Data were analyzed with SPSS for windows version 13. Descriptive statistics including means, standard deviations, and frequencies were computed. The student's t-test was used to compare means of variables. Values were considered significant if $\mathrm{P}<0.05$ (95\% CI). The value of the prediction rule were estimated for indices by means of a receiver operating characteristic (ROC) curve and the area under the curve (AUC) was reported for independent predictors.

\section{Results}

From March 2006 to May 2008, fifty consecutive cirrhotic patients (41 men, 9 women) referring to the gastroenterology clinic of AlZahra hospital, Isfahan, were enrolled in this study. Patients' demographic and clinical characteristics are shown in Table 1.

Mean age $( \pm$ SD) of these patients was 52.1 (16.2) years (range: 18-83 years). As well, the mean duration of their disease was 33.6 $( \pm 32.1)$ months (range: 1-144 months). The diagnosis of cirrhosis had been brought up for these patients using laboratory or clinical examinations or based on liver biopsy. Among the studied probable etiologies of cirrhosis (HBV, HCV, auto-immune hepatitis, $\mathrm{HBC} / \mathrm{HCV}$, probably alcohol, and idiopathic causes), HBV and idiopathic causes were most frequent in these patients (each one, $38 \%$ ). Table 2 shows the frequency percents of studied etiologies. All the studied patients underwent upper GI endoscopy. Based on our endoscopic findings, moderate to sever EV was found in 31 patients
(62\%) (Group B: 16 (32\%) were in class II, and $15(30 \%)$ were in class III) and 19 patients (38\%) had mild (6 patients; $12 \%)$ or no(13 patients; 26\%) EV (Group A). Based on the endoscopic findings and grade II was the most frequent one among our studied patients. The severity grade frequencies are shown in Table 3 . Using t-test, the means of quantitative variables (age, platelet count, serum albumin, total serum bilirubin, SGOT, SGPT, INR, Spleen diameter, Portal vein diameter, Portal vein cross section area, Splenic artery RI, hepatic artery RI, mean portal vein velocity, congestive index, Portal hypertension index, liver vascular index, and platelet count (PC)/spleen diameter (SD) ratio) were compared between groups A and B. It's shown that the means of platelet count and the laboratory amounts of total serum bilirubin, SGOT, and SGPT in group B (patients with moderate to severe EV) were meaningfully more than patients in group A (with mild or no $\mathrm{EV})(\mathrm{P}=<0.0001$,
$0.041,0.011$, and 0.035 , respectively). Also, the mean of PC/SD ratio in group B patients was meaningfully lower than its mean in group $\mathrm{A}$ $(\mathrm{P}<0.0001)$. For other quantitative diameters, no meaningful difference was found between the two mentioned groups ( $\mathrm{P}>0.05)$.

We used ROC curve to assess the predicting value of Doppler ultrasonic indices (Figure 1) and the area under the curve (AUC) was calculated for each index. Table 4 shows the measurement of AUC for these assessed indices. As we have considered the AUC $>0.7$ to be statistically significant, no one of ultrasonic indices were significant predictors for the presence of EV. Spearman regression was also used to relationship between EV grade and the Doppler ultrasonic indices. Based on our findings, portal hypertension had a direct significant relationship with $\mathrm{EV}$ severity $(\mathrm{P}=0.029)$, but $\mathrm{EV}$ grade had no significant relationship with congestion index and liver vascular index $(\mathrm{P}=0.055$ and 0.058 , respectively).

Table 1. Description of variables in two studied groups.

\begin{tabular}{lccc} 
P-value & $\begin{array}{c}\text { Group patients A: } \\
\text { without EV } \\
\text { (Mean } \pm \text { SD) }\end{array}$ & $\begin{array}{c}\text { Group patients B: } \\
\text { with EV } \\
\text { (Mean } \pm \text { SD) }\end{array}$ & Variable \\
- & 19 & 31 & Number of patients \\
$>0.05$ & $52.3(15.9)$ & $52.10(15.7)$ & Age(yrs.) \\
\hline$<0.05$ & $126000(49426)$ & $72839(31247)$ & Platelet count $\left(\mathrm{mm}^{3}\right)$ \\
$>0.05$ & $2.8(0.76)$ & $2.6(0.74)$ & Serum Albumin(g/dL) \\
\hline$<0.05$ & $3.8(3.1)$ & $7.4(7.7)$ & Total bilirubin(mg/dL) \\
$<0.05$ & $70(29.3)$ & $130(96.1)$ & SGOT(IU/dL) \\
\hline$<0.05$ & $51.8(38.2)$ & $75.7(35.8)$ & SGPT(IU/dL) \\
$>0.05$ & $1.9(0.73)$ & $2.2(1.09)$ & INR \\
\hline$<0.05$ & $131.3(24.5)$ & $151.3(30.1)$ & Spleen diameter(mm) \\
$<0.05$ & $1007.8(425.4)$ & $502.1(233.4)$ & PC/SD \\
\hline
\end{tabular}

Table 2. The frequency of studied etiologies for cirrhosis.

\begin{tabular}{lcc} 
& Number & $\%$ \\
Auto immune hepatitis & 2 & 4 \\
Unknown & 19 & 38 \\
\hline HBV & 19 & 38 \\
HCV & 7 & 14 \\
\hline HBV/HCV & 1 & 2 \\
Probably alcohol & 2 & 4 \\
\hline Total & 50 & 100 \\
\hline
\end{tabular}

Table 3. The severity grades frequencies of EVs.

\begin{tabular}{lcc} 
Grade & Number & $\%$ \\
\hline 0 & 13 & 26 \\
I & 6 & 12 \\
\hline II & 16 & 32 \\
III & 15 & 30 \\
\hline Total & 50 & 100 \\
\hline
\end{tabular}

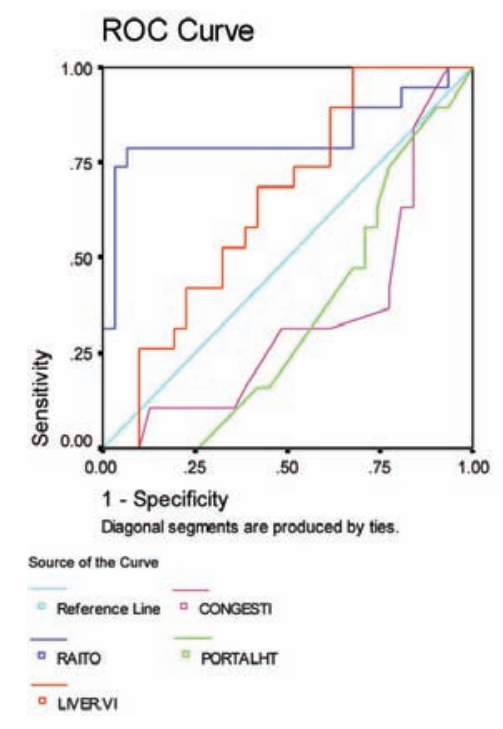

Figure 1. ROC curves for ratio, congestive index, portal Hypertensive index, and Liver vascular index. 


\section{Discussion}

Variceal gastrointestinal bleeding is one of the most common life-threatening complications of portal hypertension with significant morbidity and mortality. Variceal size is identified to be one of the most important factors responsible for first variceal hemorrhage. ${ }^{21}$ $10-20 \%$ of small varices progress in size during one year ${ }^{22}$ which is close to $20-30 \%$ risk of bleeding in first 2-year after first detection. ${ }^{23}$ It seems that recognizing patients with elevated risk of bleeding for on time interventions will reduce morbidity and financial burden in initial diagnosis or periodic intervals thereafter.

The number of patients undergoing screening for the presence of EV is likely going to increase in the near future as a result of the growing number of patients with chronic liver disease. $^{24,25}$ Consensus based guidelines recommend endoscopic screening of all cirrhotic patients for the presence of varices at the time of diagnosis. ${ }^{26}$ Relatively low risk of bleeding in compensated cirrhotic patients and a need to avoid invasive and avoidable procedures, suggest performing an upper gastrointestinal endoscopy only on those patients with clinical evidence of portal hypertension. ${ }^{27}$ Therefore, there is a particular need for non-invasive predictors of the presence of $\mathrm{EV}$ as they might help relieve medical, social, and economic costs. Meanwhile some recent studies addressed newly investigated method ,capsule endoscopy, for screening of these patients that can be an acceptable alternative in some conditions but cannot be suggested to replace UGIEs. ${ }^{28}$

We studied 50 of consecutive newly diagnosed cirrhotic patients. The mean age of studied patients was $52.1( \pm 16.2)$ and EV was present in $62 \%$ of them, which are nearly similar to some other same studies ${ }^{12,13}$ Based on the statistical findings of our study, no one of sonographic measured characters (spleen size, portal vein diameter and cross section area, resistance of hepatic and splenic arteries, Portal vein flow velocity, congestive index, portal Hypertensive index, and Liver vascular index) had acceptable predictive value for the presence of EV. As well, only the portal Hypertensive index had a significant relationship with the severity of EV and can be used in advanced assessment the patients with well proved varices.

Some other studies have reported the same results as ours. Feng-Hua L et al. have reported that Doppler ultrasonography had no value in the identification of patients with cirrhosis at risk of variceal bleeding. ${ }^{29}$ Also, Jeon SW et al. found that Doppler measurement was not helpful in distinguishing the presence of

Table 4. The amounts of AUC for assessed doppler ultrasonographic indices.

\begin{tabular}{lcccc} 
ROC curve of variables & Area under the curve & Lower & Upper & $\mathrm{P}$ \\
PC/SD & $0 / 82$ & $0 / 673$ & $0 / 967$ & $<0 / 0001$ \\
Congestive index & $0 / 34$ & $0 / 182$ & $0 / 497$ & $0 / 059$ \\
\hline Portal hypertension index & $0 / 34$ & $0 / 191$ & $0 / 489$ & $0 / 06$ \\
Liver vascular index & $0 / 647$ & $0 / 495$ & $0 / 798$ & $0 / 084$ \\
\hline
\end{tabular}

varices. However, clinical tests including biochemistry and ultrasonography would be useful in selecting eligible patients for screening endoscopy. ${ }^{30}$

However, many other studies have reported different findings, comparing with our results. Dib et al. have found that the presence of EV is related to the all hepatic and splenic hemodynamic parameters in which, the most accuracy was for splenic arterial resistance and portal Hypertensive index. ${ }^{13}$ Also, Testa et al. found a significant relationship between the congestive index and the presence of EV. ${ }^{14}$

Schepis F et al. have reported that compensated cirrhotic patients should be screened by upper gastrointestinal endoscopy when prothrombin activity less than $70 \%$, platelet count less than $100 \times 10^{9} / \mathrm{L}$, and ultrasonographic portal vein diameter greater than 13 $\mathrm{mm}$ are observed, whereas those without any of these predictors should not undergo endoscopy. They also concluded that the contribution provided by portal Doppler ultrasonographic parameters does not appear of practical utility. ${ }^{31}$ As well, Sarwar $\mathrm{S}$ et al. shown that patients with portal vein diameter $>11 \mathrm{~mm}$ are more likely to have high grade varices. ${ }^{32}$

Prihatini J et al. showed that platelet count, portal vein diameter and anteroposterior splenic measurement can be used as non invasive parameters to detect esophageal varices in cirrhotic patients. ${ }^{33}$

Tarzamni MK et al. have suggested two independent situations for beginning endoscopic evaluation of compensated cirrhotic patients: Portal hypertensive index $>2.08$ and spleen size $>15.05 \mathrm{~cm}$. These factors may help identifying patients with a low probability of LEV who may not need upper gastrointestinal endoscopy. ${ }^{34}$ Also, Chen-Hua L et al. found that splenoportal index (SPI) can serve as a useful noninvasive index to predict the presence or absence of esophageal varices. ${ }^{35}$

In summary, our data indicate that using Doppler sonographic evaluations does not allow predicting the presence of LEV with a fairly high accuracy. However, values for the non-invasive indicators from this study and comparables need to be validated in more prospective studies with greater sample sizes and better sonographic equipments.

\section{Appendix}

Authors based on present data suggest to use the Doppler assessment as an predictor of severity of varices, especially portal hypertension index . But as primary screening it seems to need more complementary laboratory indices like PC/SD diameter not to miss any probable patient with varices. We recommend to enroll more studies on the the relation of other Doppler indices with severity of varices rather than primery screening.

\section{References}

1. Hegab AM, Luketic VA. Bleeding esophageal varices. How to treat this dreaded complication of portal hypertension. Postgrad Med 2001;109:75-6,81-6,89.

2. Grace ND. Prevention of initial variceal hemorrhage. Gastroentrol Clin North Am 2003;21:149-53.

3. Smith J, Graham D. Variceal hemorrhage. Gastroentrol 1998;82:968-9.

4. Garcia-Tsao G. Current management of the complications of cirrhosis and portal hypertension: variceal hemorrhage, ascites, and spontaneous bacterial peritonitis. Gastroenterology 2001;120:726-48.

5. D'Amico G, Pagliaro L, Bosch J. The treatment of portal hypertension: a meta-analytic review. Hepatology 1995;22:332-54.

6. de FranchisR, Dell'Era A, Iannuzzi F. Diagnosis and treatment of portal hypertension. Dig Liver Dis 2004;36:787-98.

7. Graham D, Smith J. The course of patients after variceal hemorrhage. Gastroentrol 1996;80:800-7.

8. Piscaglia F, Donati G, Sella E. Value of splenic Doppler ultra sound in the diagnosis of portal hypertension. Ulterasound Med Biol 2001;27:893-9.

9. Tsokos M, Turk EE. Esophageal variceal hemorrhage presenting as sudden death in outpatients. Arch Pathol Lab Med 2002;126:1197-200.

10. Dombal FT, Clarck J, Camp S, et al. Progenspstic factors in upper GI bleedings. Endoscop 1999;18:65-9.

11. Bratovic I, Lacevic N. Management of esophageal varices. Med Arh 2002;56 
Suppl 1:11-2.

12. Korula J. Platelet count/spleen diameter ration predicted the presence of esophageal varices in liver cirrhosis. ACPJ Club 2004;140:53-80.

13. Dib N, Konate A, Oberti F, Cales F. Noninvasive diagnosis of portal hypertension in cirrhosis. Gastroenterol Clin Biol 2005;29:975-87.

14. Testa R, Testa E, Giannini F, et al. Noninvasive raito Indexes to evaluate fibrosis staging in chronic hepatitis C: role of platelet count/ spleen diameter raito index. J Inter Med 2006;260:142-50.

15. Ng FH, Wong SY, Loo CK, et al. prediction of oesophagogastric varices in patients with liver cirrhosis. J Gastroenterol Hepatol 1999;14:785-90.

16. Giannini E, Botta F, Borro P, et al. Platelet count/spleen diameter ratio: proposal and Validation invasive parameter to predict the presence of oesophageal varices in patients with liver cirrhosis. Gut 2004:53:914-5.

17. Madhotra R, Mulcahy HE, Willner I, Reuben A. Prediction of esophageal varices in patients with cirrhosis. J Clin Gastroenterol 2002;34:81-5.

18. WatanabeS, Hosomi N, Kitade Y, et al. Assessment of the presence and severity of esophagogastric varices by splenic index in patients with liver cirrhosis. J Comput Assist Tomogr 2000;24:788-94.

19. Piscaglia F, Donati G, Cecilioni L, et al. Influence of the spleen on portal haemodynamics: a non-invasive study with Doppler ultrasound in chronic liver disease and haematological disorders. Scand J Gastroenterol 2002;37:1220-7.

20. GianniniE, Botta F, Borro P, et al. Platelet count/spleen diameter ratio: proposal and validation of a non-invasive parameter to predict the presence of oesophageal varices in patients with liver cirrhosis. Gut 2003;52:1200-5.

21. Jalan R, Hayes PC. UK guidelines on the management of variceal haemorrhage in cirrhotic patients. British Society of Gastroenterology. Gut 2000;46: III1-III15.

22. Cale`s P, Desmorat H, Vinel JP, et al. Incidence of large oesophageal varices in patients with cirrhosis: application to prophylaxis of first bleeding. Gut 1990;31:1298-302.

23. Christensen E, Fauerholdt L, Schlichting P, et al. Aspects of the natural history of gastrointestinal bleeding in cirrhosis and the effect of prednisone. Gastroenterology 1981;81:944-52.

24. Metcalf M, Brown N, Peterson S, et al. Health care costs associated with chronic hepatitis B. Am J Health Syst Pharm 1999;56:232-6.

25. Wong JB, McQuillan GM, McHutchinson JG, Poynard T. Estimating future hepatitis C morbidity, mortality, and costs in the US. Am J Publ Health 2000;90:1562-9.

26. D’Amico G, Garcia-Tsao G, Cale`s P, et al. Diagnosis of portal hypertension: How and when. In: De Franchis R, ed. Proceedings of the Third Baveno International Consensus Workshop on Definitions, Methodology and Therapeutic Strategies. Oxford: Blakwell- Science; 2001. pp. 36-63.

27. Grace ND, Groszmann RJ, Garcia-Tsao G, et al. Portal hypertension and variceal bleeding: an AASLD single topic symposium. Hepatology 1998;28:868-80.

28. Guturu P, Sagi SV, Ahn D, et al. Capsule endoscopy with PILLCAM ESO for detect- ing esophageal varices: a meta-analysis. Minerva Gastroenterol Dietol 2011;57:111.

29. Feng-Hua Li, Hao J, Jian-Guo Xia, et al. Hemodynamic analysis of esophageal varices in patients with liver cirrhosis using color Doppler ultrasound. World J Gastroenterol 2005;11:4560-5.

30. Jeon SW, Cho CM, Tak WY, et al. The value of Doppler-ultrasonography and laboratory tests as noninvasive predictors of the presence of esophageal varices in patients with chronic liver disease. Korean $\mathrm{J}$ Gastroenterol 2006;48:180-7.

31. Schepis $\mathrm{F}$, Cammà $\mathrm{C}$, Niceforo $\mathrm{D}$, et al. Which patients with cirrhosis should undergo endoscopic screening for esophageal varices detection? Hepatology 2001;33:333-8.

32. Sarwar S, Khan AA, Alam A, et al. Nonendoscopic prediction of presence of esophageal varices in cirrhosis. J Coll Physicians Surg Pak 2005;15:528-31.

33. Prihatini J, Lesmana LA, Manan C, Gani RA. Detection of esophageal varices in liver cirrhosis using non-invasive parameters. Acta Med Indones 2005;37:126-31.

34. Tarzamni MK, Somi MH, Farhang S, Jalilvand M. Portal hemodynamics as predictors of high risk esophageal varices in cirrhotic patients. World J Gastroenterol 2008;14:1898-902.

35. Liu CH, Hsu SJ, Liang CC, et al. Esophageal Varices: Noninvasive Diagnosis with Duplex Doppler US in Patients with Compensated Cirrhosis. Radiology 2008;248:132-9. 